\title{
Application of subspace detection on a surface seismic network monitoring a deep silver mine
}

\author{
DJA Chambers National Institute for Occupational Safety and Health, USA
}

MS Boltz National Institute for Occupational Safety and Health, USA

JR Richardson National Institute for Occupational Safety and Health, USA

SA Finley National Institute for Occupational Safety and Health, USA

\begin{abstract}
Seismic monitoring is an important tool for understanding and mitigating seismic risk in many mining operations, especially those with deep, burst-prone conditions. However, establishing and operating a seismic network that produces quality seismic data can be very expensive due to equipment and labour costs associated with installation and maintenance of seismic stations. Researchers with the National Institute for Occupational Safety and Health are exploring data processing techniques that can improve the quality of a seismic event catalogue that do not require installing additional instrumentation. This paper presents the application of subspace methods to increase event detection capabilities of a surface seismic network monitoring a deep underground metal mine in the northwest of the United States of America. Events recorded on the network from late April to early July 2016 are used to identify similar, lower-magnitude events that occurred in a 15-day study period in June of the same year. False detection rates were evaluated by comparing results with a catalogue generated by an in-mine seismic monitoring system, and by visually examining filtered continuous waveform data at the nearest stations to the underground workings. The number of successful event detections more than doubled, with no false detections. However, detected events included production blasts that required screening based on proximity to blasting time. Acceptable estimates of magnitudes and locations for newly detected events were obtained. The application of similar methodologies to other networks may substantially augment event catalogues and provide additional data that can be used in seismic risk analysis to improve mine safety. When continuous waveform data are stored, such processing may be undertaken long after data collection is complete - a particularly valuable capability for investigating emerging stability issues.
\end{abstract}

Keywords: seismic, event detection, waveform similarity

\section{Introduction}

The interpretation of mining-induced seismicity (MIS) is often useful for meeting a variety of mine safety objectives. A common application of seismic monitoring is to use levels of seismicity as a potential indicator of stability. Areas with seismic activity greater than normal are treated with caution, and changes in seismicity levels can help determine when to allow re-entry to an affected section after a large event (Vallejos \& McKinnon 2011). Additionally, event locations can be used to identify and delineate potentially hazardous geological features. For example, Spottiswoode and Milev (1998) used waveform similarity to define planes of MIS likely corresponding to geological structure. There are also several studies that have found features in MIS data that may be precursors to large events (Lu et al. 2005; Zhao \& Jiang 2010; Kubacki et al. 2014), and other studies that demonstrate the usefulness of medium-term to long-term seismic forecasting for hazard assessment (Potvin 2009; Spottiswoode 2010). MIS can also be used in conjunction with numerical and geological methods to create more holistic mine models (e.g. Mercer \& Bawden 2005; Bewick et al. 2009).

Many types of seismic data are potentially useful for engineering and safety applications, but the most common is an event catalogue that, at a minimum, is a list of seismic event origin times, locations, and 
magnitudes (for a basic mining seismology review, see Gibowicz \& Kijko 1994). Ideally, measures of confidence or uncertainty for each of the fields are also calculated. In a mining setting, the catalogue conveys quantitative information on the ground response to extraction and, thus, mine design performance. However, in order to use a seismic catalogue for any application, it is important to ensure that the quality requirements are met. Even if the catalogue quality is adequate, improving the catalogue often leads to better analysis results. The surest strategy for improving catalogue quality produced by a given network is to deploy additional instruments that are closer to, and better surround, the source region. However, in many resource-constrained scenarios, additional installations are not feasible. In such cases, the only way to improve a catalogue is to improve the underlying data processing. Seismic data processing is complex and includes many components, but the first optimisable step in the workflow, and the focus of this study, is event detection.

\subsection{Seismic event detection and matched filters}

The first task in creating a catalogue is identifying anomalous signals (events) in data recorded by seismic instruments. Traditionally, event identification has been accomplished with amplitude threshold detectors, which require relatively high signal-to-noise ratios (SNR). Amplitude threshold detectors range in complexity from simple short-term average over long-term average algorithms to complex schemes that may apply various data transformations and have many tuneable parameters (Baer \& Kradolfer 1987; Allen 1982; Lomax et al. 2012). Frequency methods - another approach to event detection - have distinct advantages and disadvantages compared to time-domain methods and are common in many workflows (e.g. Gledhill 1985; Linville et al. 2014; Vaezi \& Van der Baan 2015). Additionally, there are many less common event detection techniques including those based on statistical distributions (Langet et al. 2014), neural networks (Wang \& Teng 1995), back projection (Kiser \& Ishii 2013), and many others. A large amount of research effort has been invested in developing and refining detection methods, and it remains a very active area of research in the seismological community (see Sharma et al. 2010 for an overview).

A well-established detection method with renewed interest is matched filtering. Conceptually, matched filters operate by scanning continuous data for signals that are similar to previously detected waveforms (referred to as training events). A simple matched filter, the cross-correlation detector, operates by quantifying the similarity between a single training event (Figure 1(a)) and continuous data potentially containing similar, low-amplitude waveforms (Figure 1(b)). The formal measure of similarity, the correlation coefficient $(c c)$, between the training event and continuous data is calculated for each time step (Figure 1(c)). Spikes in the $c c$ vector indicate potential detections. The correlation coefficient can range from -1.0 to 1.0. A value of -1.0 or 1.0 indicates that the two digitised waveforms (vectors) are identical if one of the waveforms is multiplied by a scaling factor with the same sign as the correlation coefficient. A value of 0.0 indicates maximal dissimilarity (i.e. orthogonality). On digital seismograms, the cross-correlation coefficient can be calculated in the time domain as the dot product of two demeaned, normalised vectors:

$$
c c_{i}=\frac{(x-\bar{x})}{|x-\bar{x}|} * \frac{\left(y_{i}-\bar{y}_{i}\right)}{\left|y_{i}-\bar{y}_{i}\right|}
$$

where:

$$
\begin{aligned}
c c_{i} & =\text { cross-correlation coefficient at time step } i . \\
x & =\text { training event, or vector one, of length } n . \\
\bar{x} & =\text { mean of } x . \\
y_{i} & =\text { continuous data, or vector two, of length } n \text { starting at time step } i . \\
i & =\text { current sample index. } \\
* & =\text { dot product. } \\
|x| & =\mathrm{L} 2 \text { norm of } x \text { (can be thought of as the magnitude, or length, of } x \text { ). }
\end{aligned}
$$


A: Signal from Earthquake

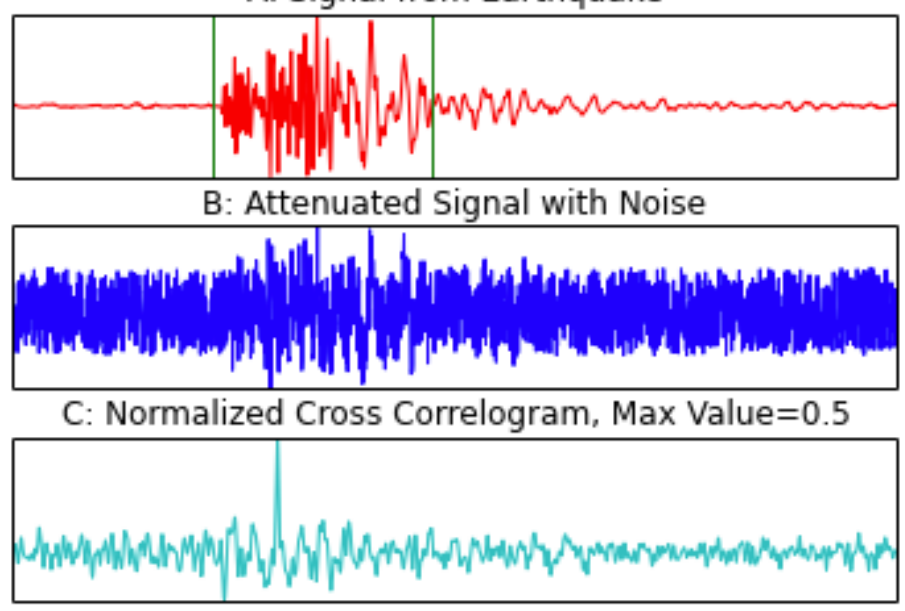

Figure 1 Conceptual explanation of cross-correlation detection. Panel A shows the waveform of a training seismic event. The waveform is trimmed by the vertical green lines to exclude most of the pre- and post-event noise. Panel B shows the signal in A embedded in normally distributed noise. Panel C is the correlation coefficient vector. After Chambers et al. (2015a)

Matched filters have been of great interest to the Comprehensive Nuclear-Test-Ban Treaty (CTBT) community, but are also particularly promising for detecting MIS, because most mines that induce significant amounts of seismicity produce a large number of events with similar waveforms (e.g. Schulte-Theis \& Joswig 1993; Spottiswoode \& Milev 1998). For this reason, and because mining events often have relatively good ground-truth, it is common for studies to use MIS to assess matched filter performance. For example, Gibbons and Ringdal (2006) used a mining seismicity dataset in demonstrating their cross-correlation detection technique, and Chambers et al. (2015b) showed that subspace detection - a generalisation of cross-correlation detection - was effective in identifying and classifying seismicity originating from an underground coal mining district using data recorded on a regional seismic network.

In this study, the methodology of Chambers et al. (2015b) is applied to data recorded on a surface seismic network in order to detect seismicity produced at a deep silver mine in the northwest of the United States of America (USA). Magnitudes are estimated based on standard deviation ratios of the recorded waveforms, and locations are approximated using a nearest-neighbour approach. The results are then validated using a catalogue produced by an in-mine microseismic system and the waveforms recorded at the closest seismic stations to the mine workings.

\subsection{The Lucky Friday mine and seismic networks}

The Lucky Friday mine is a deep silver, lead, and zinc mine located in the Silver Valley of northern Idaho (USA). The mine, which is owned by Hecla Mining Company, operates at depths between 2 and $3 \mathrm{~km}$ below the surface and has experienced various damaging seismic events. Several studies seeking to understand and mitigate rockbursts have used data collected at Lucky Friday (e.g. Blake \& Leighton 1969; Jenkins et al. 1990; White \& Whyatt 1999). Still, rockbursting remains one of the most significant hazards in deep mines.

In 2011, the mine installed a modern, in-mine, microseismic system in order to better understand and mitigate seismic risks. As of July 2016, the in-mine network consisted of $2815 \mathrm{~Hz}$ geophones and a single $4.5 \mathrm{~Hz}$ low-gain sensor. The instruments sample at $5,000 \mathrm{~Hz}$ and are located near the active stopes and in older sections of the mine to the southeast (Figure 2(a)).

In collaboration with Hecla Mining Company, National Institute for Occupational Safety and Health (NIOSH) deployed the Inter-Mountain Seismic Network (IMSN), which is primarily a surface network, over the Lucky Friday mine in 2015. As of July 2016, the IMSN consisted of five broadband stations and four accelerometers/short period stations ranging in horizontal distance from $0.2 \mathrm{~km}$ to $4 \mathrm{~km}$ away from the active stopes (Figure 2(b)). The sampling rates of the stations range from 100 to 250 samples per second. 


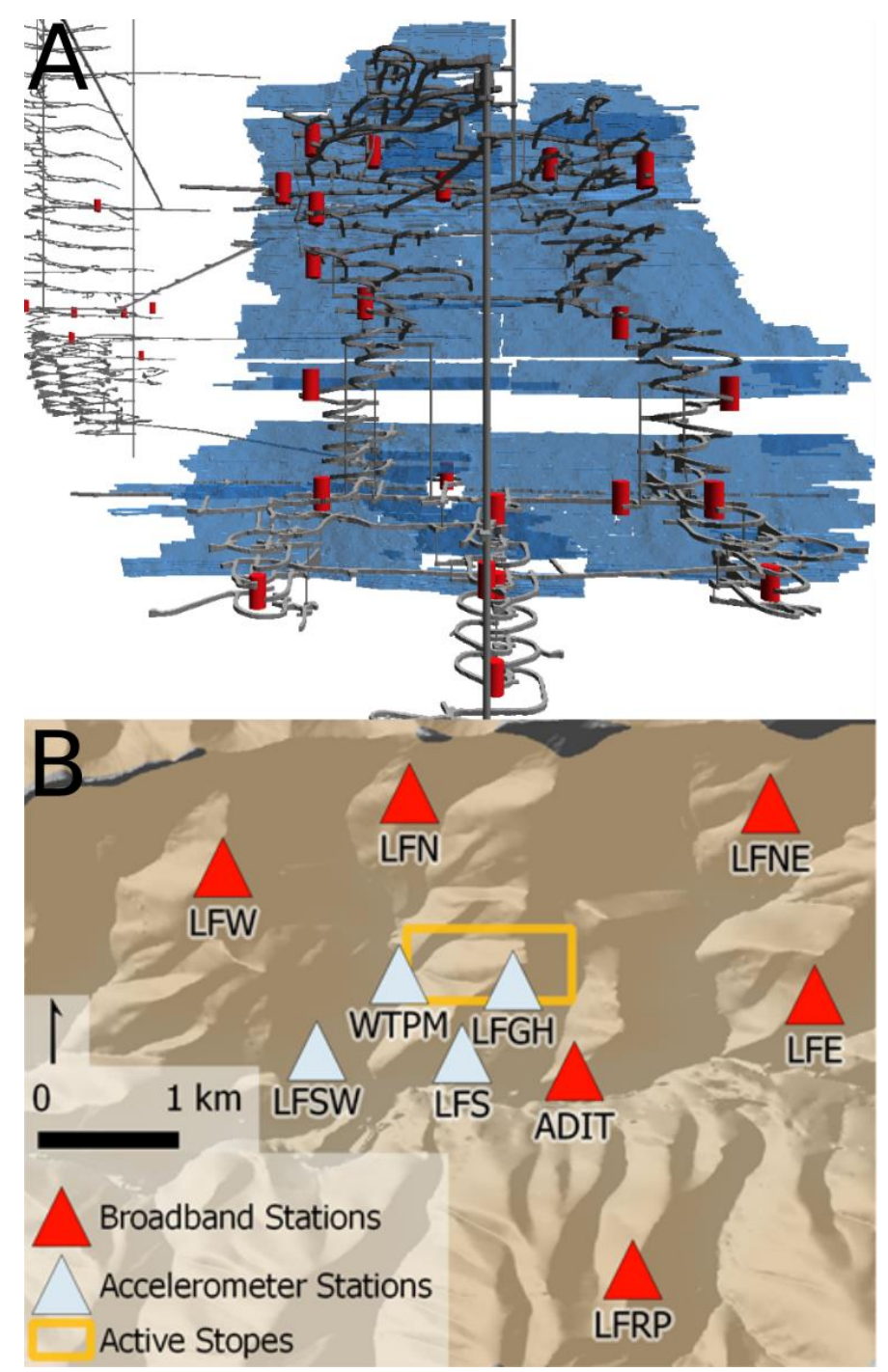

Figure 2 Panel A shows a plan view of the Inter-Mountain Seismic Network broadband stations and accelerometers/short period stations (triangles) as well as the areal extents of the active stopes (rectangle). Panel B shows a section view of the in-mine microseismic network (red cylinders), stopes (blue solids), and drifts (grey solids)

A surface network was installed by NIOSH in order to fill the gap between the in-mine seismic coverage and regional seismic coverage, which was necessary for several reasons. First, because the mine's active stopes form a near-vertical plane, with the mine workings primarily on one side of the stopes, the accessible underground areas where seismic instruments can be installed is quite limited. Consequently, determining locations and source mechanisms for larger events that tend to occur some distance from the mine workings can be difficult using the in-mine network alone. The surface stations, which can be more easily distributed horizontally, help increase focal sphere sampling and constrain locations of such events. Second, most in-mine sensors are not suitable for large event analyses that require a moderate source-receiver separation, favourable low-frequency instrument responses, and unclipped waveforms (e.g. time-domain moment-tensor inversion). Third, the two networks, when operated independently, can provide verification of detection, locations, and magnitudes produced by the other network as well as provide a backup if one network goes offline. Ultimately, the separation of the two networks is largely an artefact of the collaboration agreement between Hecla and NIOSH and the differences in data processing software used by each network. Data from both networks are periodically combined to form an authoritative catalogue. 


\section{Data}

Continuous data have been recorded by the IMSN since February 2015, with the majority of stations deployed by January 2016, although additional installations are pending. The IMSN catalogue was produced using a workflow typical of the seismic monitoring framework Earthworm (Earle et al. 2003). For event detection, the configurable amplitude threshold detector described in Allen (1982), as implemented in the 'pick_ew' module, was used to declare triggers on each station, and the module 'binder_ew' (Dietz 2002) was used to associate triggers into coherent events consistent with a preliminary velocity model. The parameters for these modules have not been formally optimised, but were selected based on values that have worked well on other microseismic networks. During this time period, many low SNR events were detected that had high arrival time uncertainties, thus, indicating that the event detection component of the data processing was adequate. After automatic processing, analysts manually inspected every event, adjusted phase arrivals and amplitude picks, and recalculated locations and magnitudes when necessary.

The in-mine microseismic catalogue was created using proprietary software that also employs an amplitude threshold detector to identify events. Histograms of the 482 events in the IMSN catalogue and the 9,872 events in the microseismic catalogue from 1 January 2016, through 30 June 2016, are shown in Figures 3(a) and 3(b), respectively. It should be noted that the microseismic catalogue uses moment magnitudes $\left(M_{w}\right)$, whereas the IMSN uses local magnitudes $\left(M_{L}\right)$ because the high frequency content of smaller events (often necessary for determining corner frequency) is greatly attenuated before reaching IMSN stations. Training events were compiled from the IMSN during the time period of 25 April to 30 June 2016, when no significant changes were made to the network. Subspace detection was applied to continuous data recorded by the IMSN from 3 June to 18 June 2016. During this time window, 805 events were recorded by the microseismic system, which were used as the verification catalogue for assessing performance of the subspace detector. All of the 805 events were automatically located and have been classified as a blast or MIS by an analyst. Only the phase picks of events with $M_{w} \geq 0.3$ have been manually adjusted and all effected events were relocated.
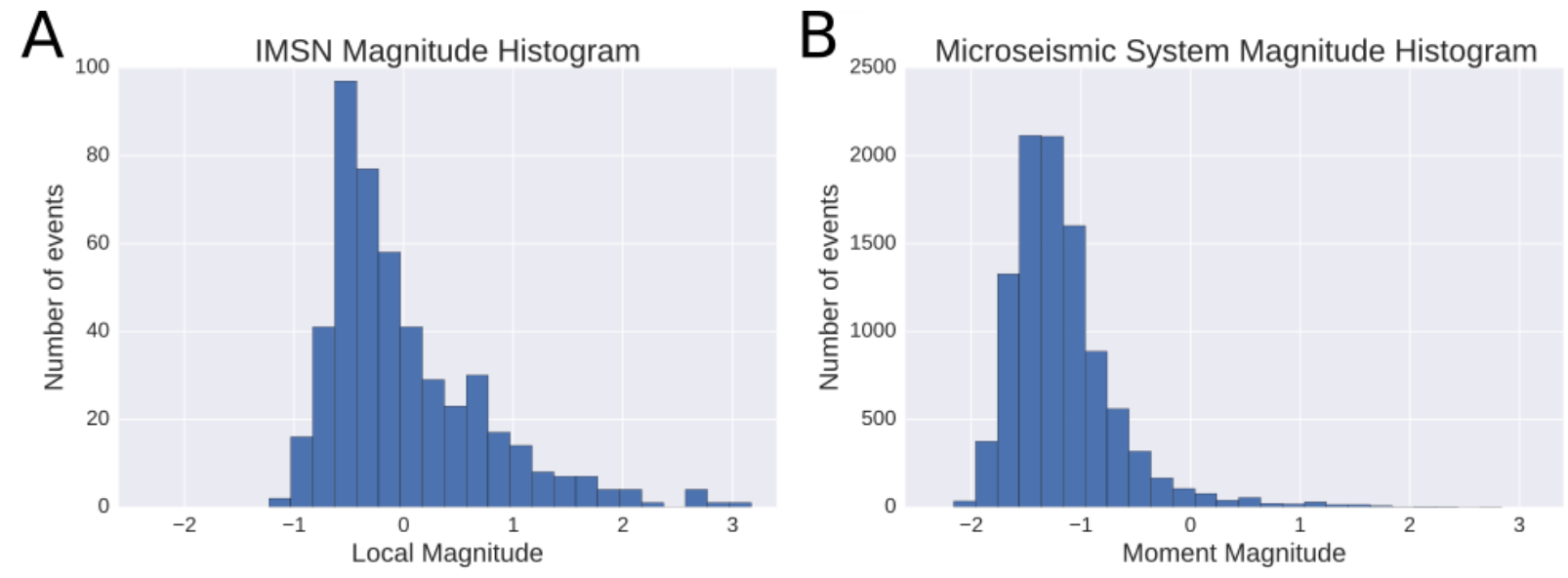

Figure 3 Magnitude histograms for events recorded by the: (a) IMSN; and, (b) in-mine microseismic system

\section{Methodology}

The subspace method entails identifying groups of events with similar waveforms, creating an orthonormal base (subspace) for each group, then scanning continuous data looking for low-amplitude signals that are similar to any of the training events in the groups. The subspace detection workflow presented in Chambers et al. (2015b), based on the procedure outlined by Harris (2006), was applied on data collected by the IMSN in order to detect events with low SNR. Additionally, magnitudes of detected events were estimated using waveform scaling, and preliminary event locations were approximated as the location of the best correlated training event. 


\subsection{Event waveform similarity clustering}

A pool of training events was identified by examining the waveforms of events in the IMSN catalogue that occurred from 25 April to 30 June 2016. These events were identified by amplitude threshold detectors applied on IMSN data. The events containing blasting waveforms were not used as training events in an effort to avoid contaminating the subspaces with non-MIS waveform signatures. The training set contained 37 events, though several of the stations did not record the entire set. The training events were then trimmed to 15 seconds duration to include all of the visible event phases and several seconds of additional data before and after the event. The channels of each station were multiplexed into a single stream in order to produce a one-dimensional (1D) vector of each training event. The waveform similarity between each pair of multiplexed training events were calculated by zero-padding one of the waveforms with several seconds at the beginning and end, applying Equation 1, and determining the maximum of the resulting correlation coefficient vector $(c c)$, (Figure 4(a)). A single-link hierarchical clustering algorithm (Harris 2006) was then used to form waveform similarity groups having cross-correlation coefficients greater than 0.4 , which is equivalent to dissimilarity value less than 0.6 (Figure 4(b)). A dissimilarity limit of 0.6 was determined to reasonably optimise grouping while still excluding very dissimilar waveforms.
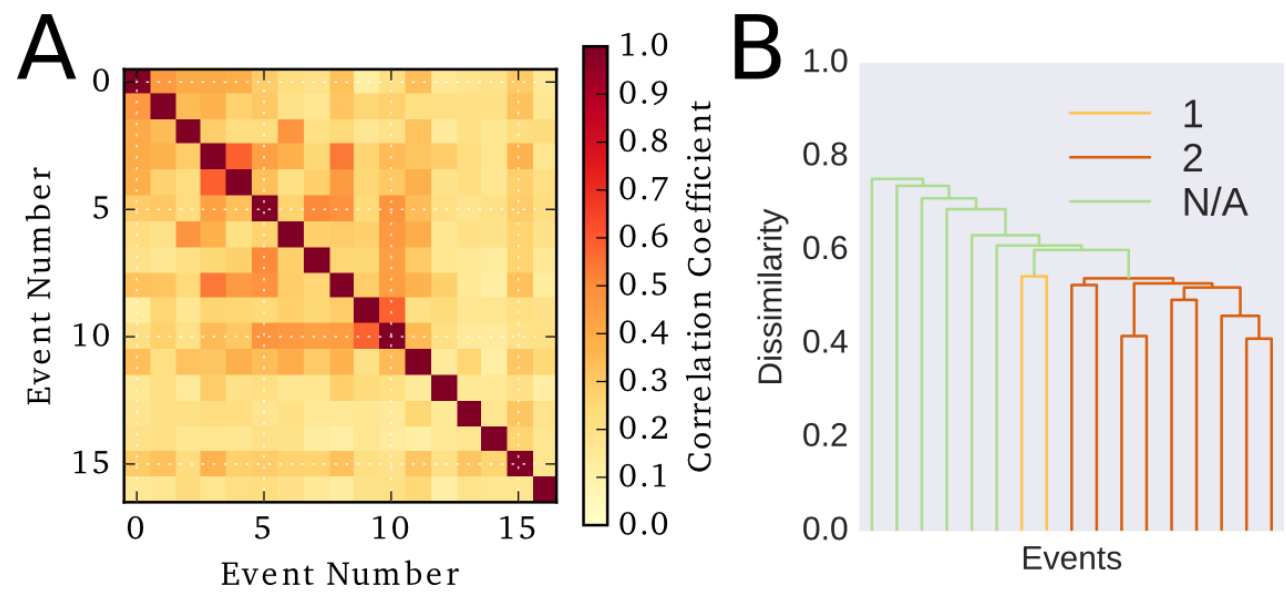

Figure 4 Example of waveform similarity clustering. Panel A shows the maximum correlation coefficient between each event pair for station LFE. Panel B shows the result of the single-link hierarchical clustering on dissimilarity (one minus the correlation coefficient, y axis)

\subsection{Subspace construction}

The next phase involves creating mathematical bases (subspaces) that contain all linear combinations of the training events, but do not contain all arbitrary vectors of the same length. The subspaces were created from each waveform similarity group by first aligning the multiplexed waveforms in order to optimise the sliding correlation coefficient (Equation 1) according to the procedure defined in Appendix B of Harris (2006). A window of 2.0 seconds was then defined around the aligned waveform group (Figure 5(a), dotted vertical lines) in order to form an $n$ (number of samples) by $m$ (number of events) matrix. Singular value decomposition (SVD), (see Poole 2014 for review) was then performed on the waveform matrix to calculate an $n$ by $m$ orthonormal basis, or subspace, $(U)$ which is said to be of $m$ dimensions. A vector (e.g. training event or segment of continuous data) can then be projected on $U$ :

$$
\boldsymbol{v}_{p}=U U^{T} \boldsymbol{v}
$$

where:

$$
\begin{aligned}
& \boldsymbol{v}_{p} \quad=\text { projected vector. } \\
& U \quad=\text { orthonormal basis of waveform group (subspace). } \\
& \boldsymbol{v} \quad=\text { vector of length } n \text { to be projected on } U . \\
& T \quad=\text { transpose operation. }
\end{aligned}
$$




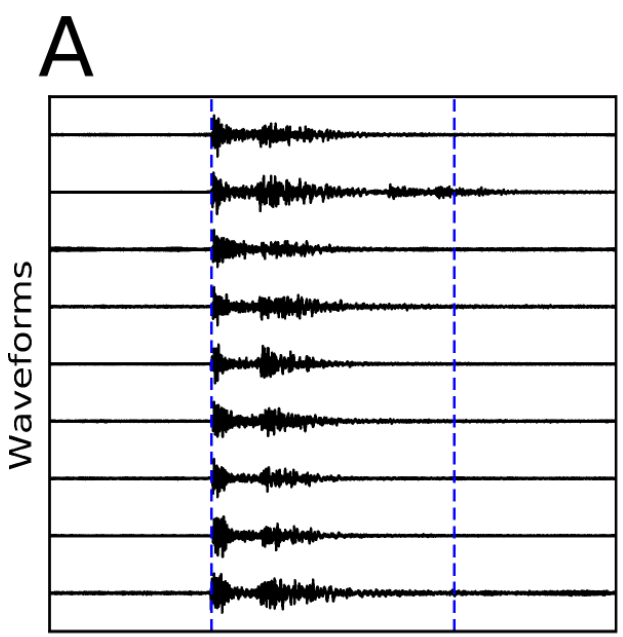

Multiplexed Time

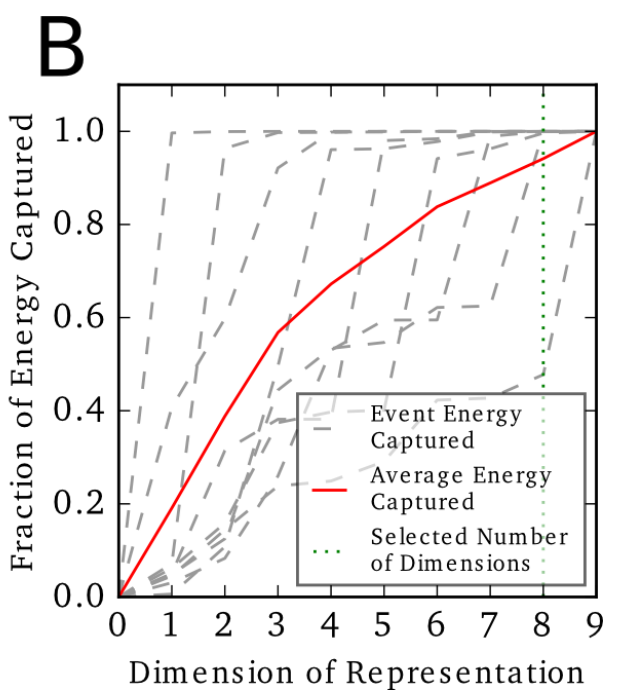

Figure 5 Subspace construction for LFE subspace two. Panel A shows the trimmed, multiplexed, and aligned waveforms defined between the dashed vertical lines. Panel B shows the subspace dimensionality determination for the same group of events with the fractional energy capture for each training event (grey dashed line), the average fractional energy captured (red line), and selected threshold (vertical dotted line)

The projected vector $\left(\boldsymbol{v}_{p}\right)$ is a representation of original vector $(\boldsymbol{v})$ in the subspace $(U)$. This is analogous to projecting a 3D vector on a plane; if the vector is nearly parallel to the plane, the projected vector is similar in magnitude (length) to the original vector. However, if the vector is nearly orthogonal to the plane, the projected vector has a much smaller magnitude than the original vector. Applying this concept, a formal measure of how similar $v$ is to the training events used to form a subspace, or how well $v$ 'fits' into the subspace, can be calculated as a ratio of the projected vector energy over the original vector energy where the term 'vector energy' simply refers to the square of the L2 norm:

$$
c=\frac{\boldsymbol{v}_{p}^{T} \boldsymbol{v}_{p}}{\boldsymbol{v}^{T} \boldsymbol{v}}
$$

where:

$c=$ fraction of vector energy captured (or fractional energy) if $v$ is a training event, or event-detection statistic otherwise.

For a 1D subspace the detection statistic $(c)$ is the square of the correlation coefficient $(c c)$.

Equation 3 was then used to reduce the dimensionality of each subspace by calculating the minimum number of subspace dimensions needed to adequately represent the constitutive waveforms. This was done by varying the number of left singular vectors (the most significant basis vectors) belonging to $U$ from 0 to $m$ and calculating the fractional energy captured $\left(c_{a v}\right)$ of each of the training events (Equations 2 and 3 ). The average fractional energy captured was then calculated for dimensions of $U\left(U_{\mathrm{dim}}\right)$ from 0 to $m$ and declared sufficient for the lowest value for which $c_{a v} \geq 0.9$. The fractional energy capture threshold of 0.9 was selected based on past experience, but can be lowered for greater dimensionality reduction, and consequently faster runtimes, or raised for potentially higher detection rates and slower runtimes. However, it should be noted that too many dimensions can increase the detectors tendency to make false detections. A sound strategy is to select a value that accounts for most of the energy in the waveform group but that also allows for some dimensionality reduction. Figure $5(\mathrm{~b})$ shows the process for station LFE subspace two, which is composed of events from group 2 of Figure 4(b), where an eight-dimensional subspace was selected. Although less than the maximum dimension of representation, which is the total number of training events in the group, the algorithm selecting eight dimensions means that most of the subspace's basis vectors were required to capture an acceptable percentage (90\% on average) of the training events' energy. A greater dimensionality reduction would be expected if the training events were more similar to one another. 


\subsection{Threshold determination and subspace detection}

The required detection statistic $\left(c_{r e q}\right)$ for declaring new detections was determined for each subspace on each station by calculating $c$ for each time step in 100 hours of randomly selected continuous data. A beta distribution was then fit to the resulting values via the maximum likelihood method. Using the beta distribution, the detection statistic required to produce a probability of false detection of $10^{-6}$ was determined. As noted by other authors (Slinkard et al. 2014), the tails of the distribution are generally a poor fit but requiring detections to occur on multiple stations can effectively cull false positives by constraining the source location.

The subspace detector was then run on all available continuous data from each station for the time period of 3 June to 18 June 2016. The resulting station detections were associated into events using a simple coincidence filter that required each detection to occur near a theoretical arrival time on at least four stations. The detections that did not meet this requirement were discarded.

\subsection{Magnitude estimation}

When a detection was declared, a waveform amplitude ratio scheme was used to estimate magnitudes. The estimation is a correlation-coefficient weighted average of the standard deviation of the detected waveform and each training event of the subspace that declared the detection:

$$
m_{i}=\frac{\sum_{j=0}^{J}\left(\log _{10}\left(\operatorname{std}\left(\boldsymbol{c}_{i}\right) / s t d\left(\boldsymbol{t}_{j}\right)\right)+m_{j}\right) c c_{i j}{ }^{2}}{\sum_{j=0}^{J} c c_{i j}{ }^{2}}
$$

where:

$$
\begin{aligned}
m_{i} & =\text { estimated magnitude for detected event } i . \\
\boldsymbol{c}_{i} & =\text { waveform of detected event } i . \\
\boldsymbol{t}_{j} & =\text { training event } j \text { belonging to the subspace which made the detection. } \\
m_{j} & =\text { reported magnitude of training event } j . \\
c c_{i j} & =\text { maximum correlation coefficient between training event } j \text { and detected event } i .
\end{aligned}
$$

Equation 4 is similar to the methodology used by Schaff and Richards (2014). It deviates from other magnitude estimation methods often used in matched filters studies (e.g. Gibbons \& Ringdal 2006) in that it accounts for energy that is dissimilar to the training events. The standard deviation method generally performs better when the SNR is not too low because magnitude estimates tend to saturate around the background noise level (SNR $\approx 1$ ). For such events, the projected energy methods would likely perform better (Chambers et al. 2015b).

\subsection{Event location approximation}

Standard methods for locating small events found through subspace detection are often not viable as the first arriving phases tend to be below the noise level, even with aggressive filtering. Because first arrival phase picks are often not possible, a nearest-neighbour approach was adopted by simply assigning the location of the newly detected event to the location of the best correlated training event (the preferred training event). If the preferred training event varied for a single detection across the stations, the location of the preferred training event selected by the most stations was used. This approximation for location often works well because events that produce similar waveforms have small inter-event separations and comparable source mechanisms (Baisch et al. 2008). The disadvantage, however, is that the locations of newly detected events are limited to the locations of the training events. Also, because waveform similarity is sensitive to source mechanism and signal amplitude, in addition to location, the preferred training event is not always the closest training event to the detected event. 
Hypocentres approximated with the preferred training event method provide reasonable starting locations for iterative location techniques. Double-difference methodologies (Waldhauser \& Ellsworth 2000) are common for locating groups of similar events with low SNR because relative lag times, estimated with cross-correlation (Schaff et al. 2004), can be used as inputs to the location method rather than absolute arrival times. Even if double difference methods fail, however, using the location of the preferred training event yields a hypocentre estimate for an event that may not be locatable otherwise.

\section{$4 \quad$ Results}

Including the 24 events already in the IMSN catalogue, there were a total of 63 verified detections. The detector returned 79 events that were not in the microseismic catalogue, which represent potential false positives. There were 742 events in the microseismic catalogue that were not detected by the subspace detector, although the majority of these had $\mathrm{M}_{\mathrm{w}} \leq-0.5$. For the verified events, the magnitude estimation performed fairly well, and can be improved by applying the relationship between estimated magnitude and microseismic moment magnitude found through orthogonal regression. The preferred training event locations provide a reasonable estimation for most events, but more stringent location methods may be needed depending on the data application.

\subsection{Verified and missed detections}

The subspace detector identified 39 events not contained in the IMSN catalogue that were found in the microseismic catalogue. Figure 6 shows example waveforms of an event detected with subspace methods that was not detected with the amplitude threshold detector, as well as the detection's preferred training event. The subspace detector more than doubled the original 24 events in the IMSN catalogue for the test period. A total of about $8 \%$ of events recorded on the microseismic system were detected with the surface stations, with a strong tendency towards higher magnitudes (Figure 7).

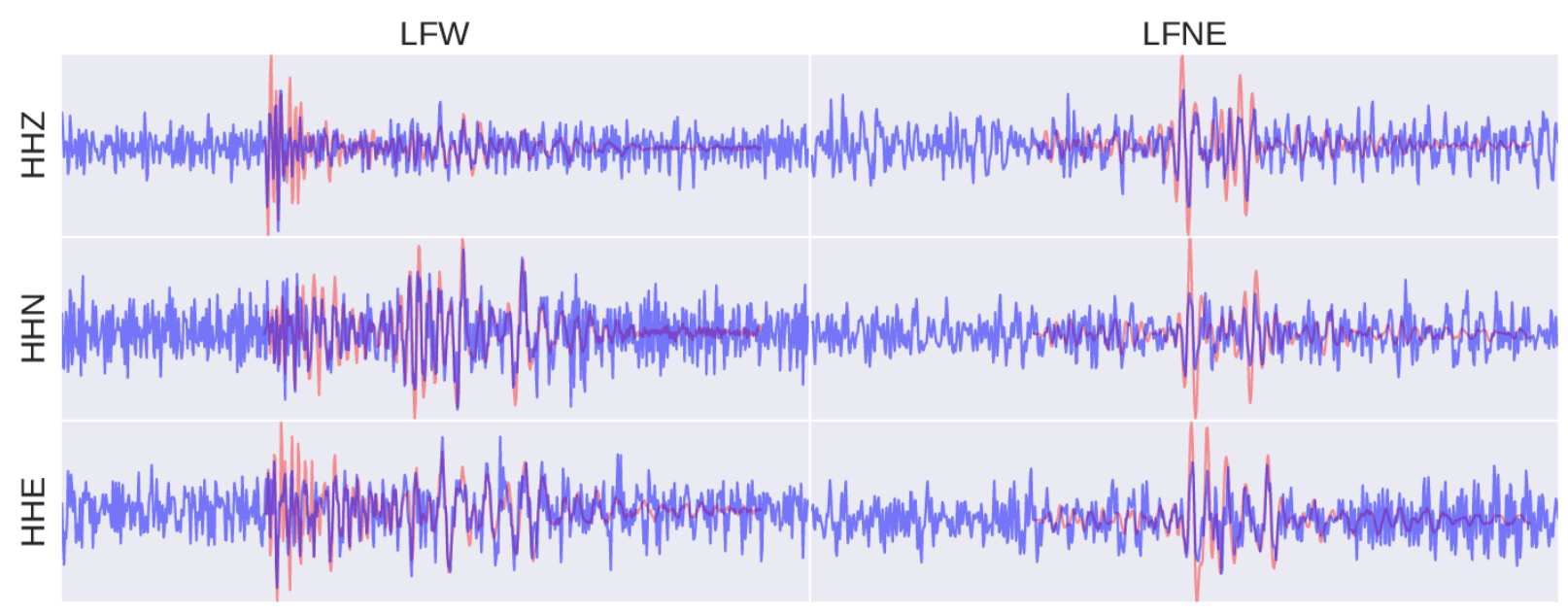

Figure 6 Three-second window showing an event identified by the subspace detector but missed by the amplitude threshold detector with the scaled-down preferred training event superimposed (red trace) 


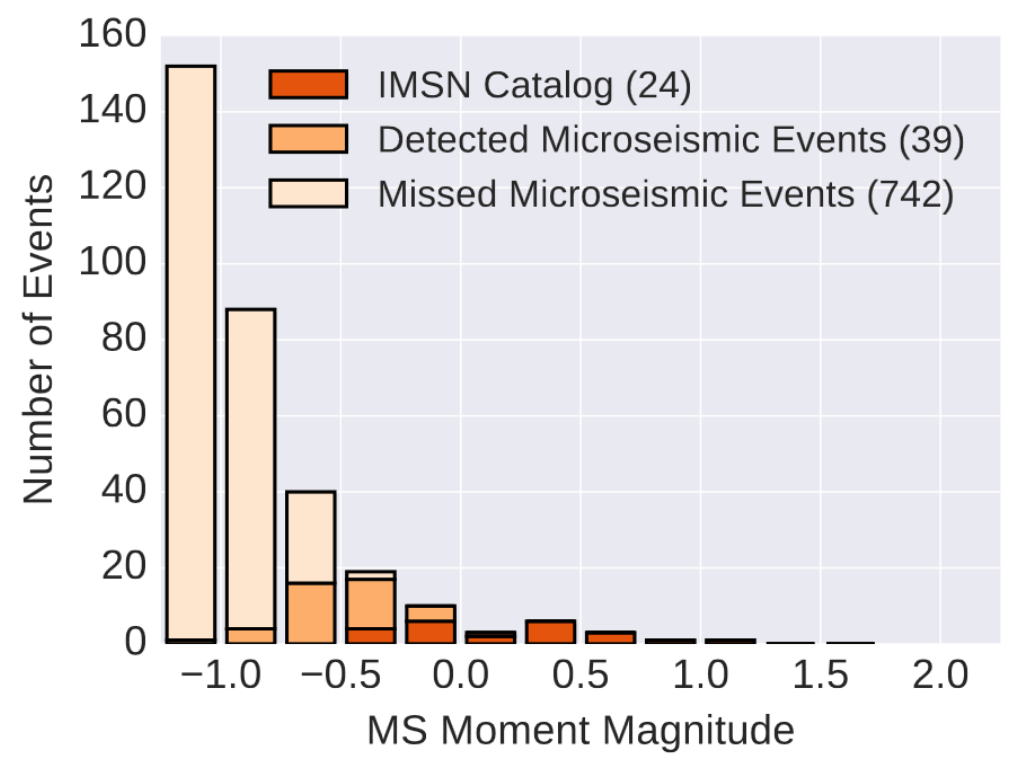

Figure 7 Detection results. Thirty-nine events were identified using subspace detection in addition to the original 24 IMSN events. Seven hundred forty-two microseismic events were not detected (events with $\mathrm{M}_{\mathrm{w}} \leq-1.5$ are not shown in the histogram)

\subsection{Unverified detections}

There were 79 detected events that were not found in the microseismic catalogue. All but eight of these, however, occurred within one second of a recorded blast (Figure 8). The high temporal proximity indicates that the majority of potential false positives are production blasts, or induced events occurring close in time to a production blast that the in-mine system did not detect or misclassified. The waveforms of the eight events not within one second of a blast were manually examined, at the closest stations, and were determined to be genuine events not found in the microseismic catalogue (Figure 9).

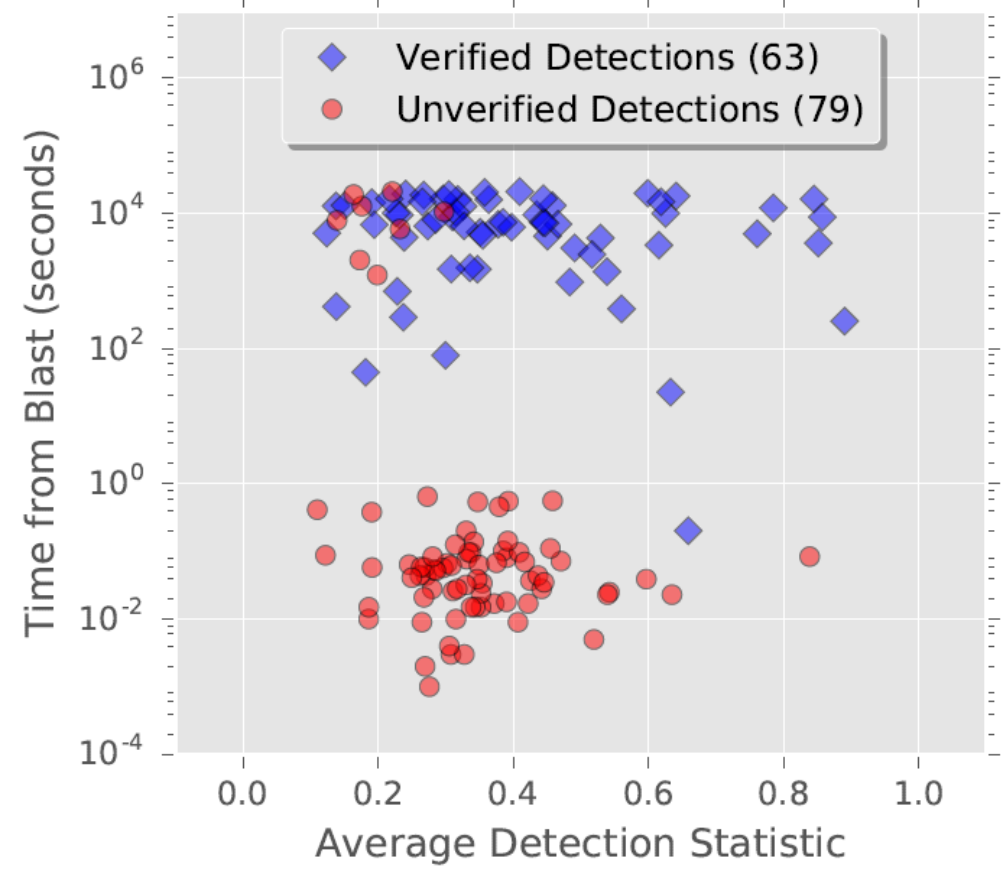

Figure 8 Average detection statistic ( $x$ axis) and distance in time to the nearest blast ( $y$ axis) of potential false detection (dots) and verified detections (diamonds) 


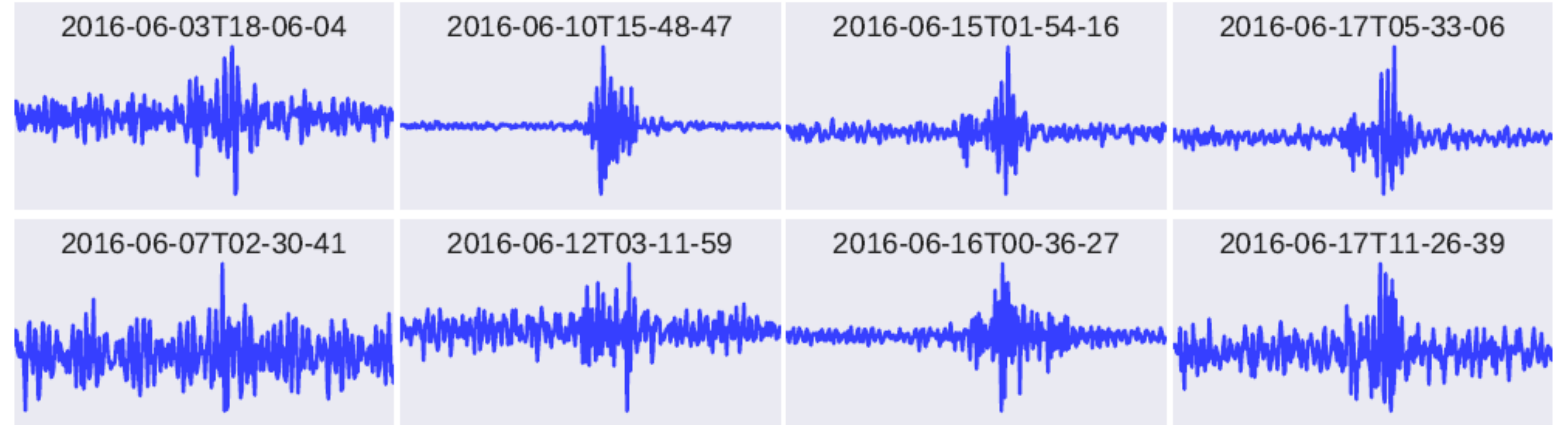

Figure 9 Waveforms of unverified detections that did not occur within one second of a production blast recorded by station WTPM. Event-like waveforms indicate that some genuine events detected by the IMSN were not detected by the microseismic system

A conservative approach would be to discard any detections within one second of a blast. However, it may be possible to discriminate between the waveforms of induced seismic events occurring at nearly the same time as a blast and the waveforms of the blast itself. For example, some of the unverified detections in Figure 7 have detection statistic values greater than 0.8 , indicating a very high degree of similarity to the training MIS events. Because it is unlikely that blast waveforms would be so similar to MIS waveforms, events with high detection statistic values are probably MIS-induced by blasting. However, the events with lower detection statistic values could be blasts, MIS events with low amplitudes, or MIS events with waveforms not well captured by any of the subspaces. Discriminating between these populations is an interesting topic for future research that could allow for identifying many events that would not be detectable using amplitude threshold detectors alone.

\subsection{Magnitude evaluation}

The majority of the estimated magnitudes for the detected events (Equation 4) were lower than the magnitudes recorded in the microseismic catalogue. However, using orthogonal regression, a linear relationship was derived to relate the estimated magnitudes to the microseismic moment magnitudes (Figure 10). The regression fit the data very well, with an $R^{2}$ value of 0.93 . When the linear equation was applied to transform the estimated magnitudes into microseismic moment magnitudes, the median residual is only 0.05 magnitude units.

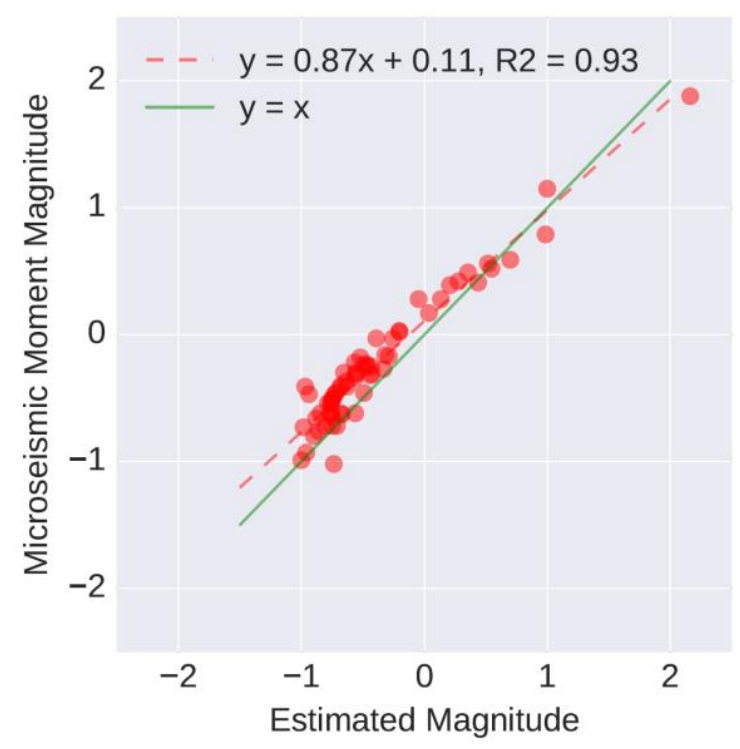

Figure 10 Estimated magnitudes (Equation 4, $\mathrm{x}$ axis) and moment magnitude in microseismic catalogue (y axis) with corresponding regression line (dotted red line) 


\subsection{Hypocentre approximation evaluation}

The nearest-neighbour hypocentre approximation performed moderately well with a median hypocentral and epicentral offset of 32 and $28 \mathrm{~m}$, respectively, between the estimated location and the location calculated by the in-mine microseismic system (Figure 11). If more training events were used, the accuracy of the event locations would increase because there would be more possible locations for the detections to adopt. For most detected events, the preferred training event was the closest training event, but for nine of the 39 newly detected events this was not the case. Setting preliminary locations of detected events in this way can serve as a reasonable starting point for iterative location algorithms that require initial locations, or as a coarse way to track energy release of repeating sources.

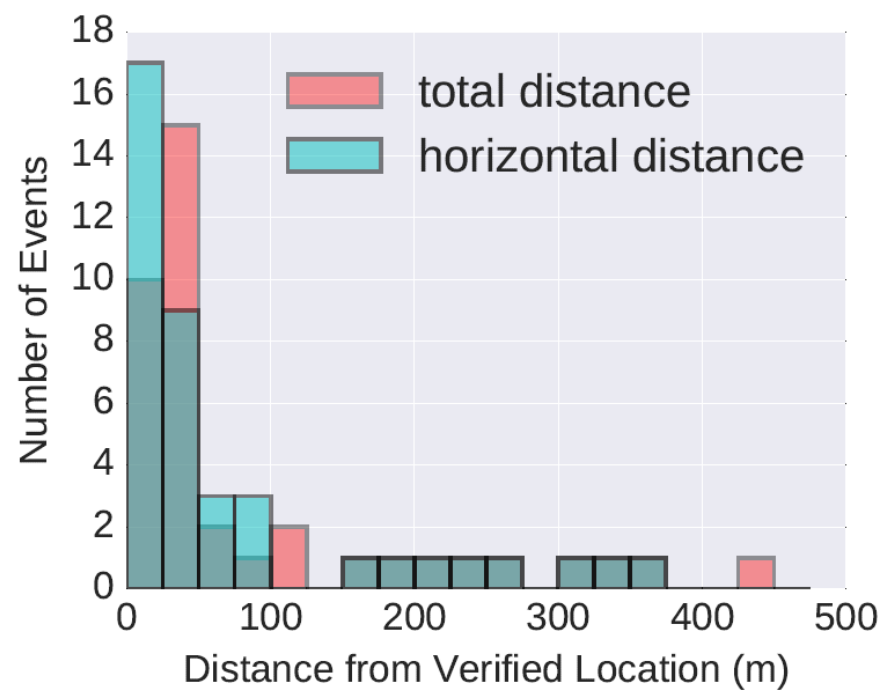

Figure 11 Histogram of total (blue) and horizontal (red) location error using the preferred training event approximation

\section{Conclusion}

The application of subspace detection increased the number of verified MIS events detected by the surface network from 24 to 63 over a 15-day trial period. This increase in detection rate was achieved through data processing techniques alone and did not require any additional instrumentation. When compared to the total number of MIS events identified by the in-mine microseismic system, however, only about $8 \%$ of the events were detected. Although the surface network will likely never perform as well as the in-mine system at event detection, it was able to identify eight events that were missed by the in-mine system, demonstrating that there are some events more amenable to successful detection by the surface network using subspace detection.

The estimated magnitudes of the detected events related very well to moment magnitudes estimated by the in-mine microseismic network after applying the transformation determined through orthogonal regression. Without calibration to the verification catalogue, however, the predicted magnitudes are internally consistent and, although slightly biased, would still be useful for many applications. By setting the detected event location to the location of the preferred training event, a reasonable hypocentre estimate for the newly identified events was achieved. For very small events, the preferred training event hypocentre approximation may be the only way to estimate location if other methods fail.

Although subspace detectors were applied exclusively to a surface network in this study, subspace techniques would likely prove effective for augmenting the detection capabilities of in-mine microseismic systems as well. With additional research, subspace methods could also be used for detecting seismic events induced immediately after blasting that amplitude threshold detectors tend to miss. The results from a subspace detector could also be used to optimise and evaluate other detection methods. Therefore, subspace detection can meaningfully complement traditional seismic data processing workflows, resulting in higher quality datasets that can be used to better understand seismic hazards and improve mine safety. 


\section{Acknowledgement}

The authors would like to acknowledge Hecla Mining Company for all its efforts and willingness to collaborate with NIOSH. The open-source seismology package ObsPy (Beyreuther et al. 2010; Megies et al. 2011) was used extensively in this research.

\section{Disclaimer}

The findings and conclusions in this report are those of the author(s) and do not necessarily represent the views of the National Institute for Occupational Safety and Health (NIOSH). Mention of any company or product does not constitute endorsement by NIOSH.

\section{References}

Allen, R 1982, 'Automatic phase pickers: their present use and future prospects', Bulletin of the Seismological Society of America, vol. 72, no. 6B, pp. 225-242.

Baer, M \& Kradolfer, U 1987, 'An automatic phase picker for local and teleseismic events', Bulletin of the Seismological Society of America, vol. 77, no. 4, pp. 1437-1445.

Baisch, S, Ceranna, L \& Harjes, H 2008, 'Earthquake cluster: What can we learn from waveform similarity?', Bulletin of the Seismological Society of America, vol. 98, no. 6, pp. 2806-2814.

Bewick, R, Valley, B, Runnalls, S, Whitney, J \& Krynicki, Y 2009, 'Global approach to managing deep mining hazards', in M Diederichs \& G Grasselli (eds), Proceedings of the 3rd CANUS Rock Mechanics Symposium, vol. 1, Canadian Rock Mechanics Association, American Rock Mechanics Association, Alexandria, pp. 12.

Beyreuther, M, Barsch, R, Krischer, L, Megies, T, Behr, Y \& Wassermann, J 2010, 'ObsPy: A Python toolbox for seismology', Seismological Research Letters, vol. 81, no. 3, pp. 530-533.

Blake, W \& Leighton, F 1969, 'Recent developments and applications of the microseismic method in deep mines', The 11th US Symposium on Rock Mechanics, American Rock Mechanics Association, Alexandria.

Chambers, D, Wempen, J, McCarter, M, Pankow, K \& Koper, K 2015a, 'Correlation of newly detected mining induced seismicity with subsidence in a Wyoming mining district', 2015 SME Annual Conference and Expo and CMA 117th National Western Mining Conference - Mining: Navigating the Global Waters, Society for Mining, Metallurgy and Exploration, Englewood, pp. 192-198.

Chambers, D, Koper, K, Pankow, K \& McCarter, M 2015b, 'Detecting and characterizing coal mine related seismicity in the Western US using subspace methods', Geophysical Journal International, vol. 203, no. 2, pp. 1388-1399.

Dietz, L 2002, 'Earthworm Module: binder_ew', viewed 17 August 2016, http://love.isti.com/trac/ew/wiki/binder_ew

Earle, P, Bittenbinder, A, Bogaert, B \& Johnson, C 2003, 'Tune to the worm: Seismic network operation using the USGS Earthworm system', Observations and Research Facilities for European Seismology, Orfeus Newsletter, vol. 5, no. 1.

Gibbons, S \& Ringdal, F 2006, 'The detection of low magnitude seismic events using array-based waveform correlation', Geophysical Journal International, vol. 165, no. 1, pp. 149-166.

Gibowicz, SJ \& Kijko, A 1994, An introduction to Mining Seismology, Academic Press, New York.

Gledhill, K 1985, 'An earthquake detector employing frequency domain techniques', Bulletin of the Seismological Society of America, vol. 75 , no. 6, pp. 1827-1835.

Harris, D 2006, Subspace Detectors: Theory, United States Department of Energy.

Jenkins, F, Williams, T \& Wideman, C 1990, 'Rock burst mechanism studies at the Lucky Friday Mine', The 31th US Symposium on Rock Mechanics (USRMS), American Rock Mechanics Association, Alexandria.

Kiser, E \& Ishii, M 2013, 'Hidden aftershocks of the 2011 Mw 9.0 Tohoku, Japan earthquake imaged with the back projection method', Journal of Geophysical Research: Solid Earth, vol. 118, no. 10, pp. 5564-5576.

Kubacki, T, Koper, K, Pankow, K \& McCarter, M 2014, 'Changes in mining-induced seismicity before and after the 2007 Crandall Canyon Mine collapse', Journal of Geophysical Research: Solid Earth, vol. 119, no. 6, pp. 4876-4889.

Langet, N, Maggi, A, Michelini, A \& Brenguier, F 2014, 'Continuous Kurtosis-based migration for seismic event detection and location, with application to Piton de la Fournaise Volcano, La Réunion', Bulletin of the Seismological Society of America, vol. 104, no. 1, pp. 229-246.

Linville, L, Pankow, K, Kilb, D \& Velasco, A 2014, 'Exploring remote earthquake triggering potential across EarthScopes' Transportable Array through frequency domain array visualization', Journal of Geophysical Research: Solid Earth, vol. 119, no. 12, pp. 8950-8963.

Lomax, A, Satriano, C \& Vassallo, M 2012, 'Automatic picker developments and optimization: FilterPicker-A robust, broadband picker for real-time seismic monitoring and earthquake early warning', Seismological Research Letters, vol. 83, no. 3, pp. 531-540.

Lu, C, Mai, YW \& Xie, H 2005, 'A sudden drop of fractal dimension: a likely precursor of catastrophic failure in disordered media', Philosophical Magazine Letters, vol. 85, no. 1, pp. 33-40.

Megies, T, Beyreuther, M, Barsch, R, Krischer, L \& Wassermann, J 2011, 'ObsPy-What can it do for data centers and observatories?', Annals of Geophysics, vol. 54, no. 1, pp. 47-58.

Mercer, R \& Bawden, W 2005, 'A statistical approach for the integrated analysis of mine-induced seismicity and numerical stress estimates, a case study-Part I: developing the relations', International Journal of Rock Mechanics and Mining Sciences, vol. 42, no. 1, pp. 47-72. 
Poole, D 2014, Linear Algebra: A Modern Introduction, Cengage Learning.

Potvin, Y, 2009, 'Strategies and tactics to control seismic risks in mines', Journal of the Southern African Institute of Mining and Metallurgy, vol. 109, no. 3, pp. 177-186.

Schaff, D \& Richards, P 2014, 'Improvements in magnitude precision, using the statistics of relative amplitudes measured by cross correlation', Geophysical Journal International, vol. 197, no. 1, pp. 335-350.

Schaff, D, Bokelmann, G, Ellsworth, W, Zanzerkia, E, Waldhauser, F \& Beroza, G 2004, 'Optimizing correlation techniques for improved earthquake location', Bulletin of the Seismological Society of America, vol. 94, no. 2, pp. 705-721.

Schulte-Theis, H \& Joswig, M 1993, 'Clustering and location of mining induced seismicity in the Ruhr basin by automated master event comparison based on dynamic waveform matching', Computers \& Geosciences, vol. 19, no. 2, pp. 233-241.

Sharma, B, Kumar, A \& Murthy, V 2010, 'Evaluation of seismic events detection algorithms', Journal of the Geological Society of India, vol. 75, no. 3, pp. 533-538.

Slinkard, M, Schaff, D, Mikhailova, N, Heck, S, Young, C \& Richards, P 2014, 'Multistation validation of waveform correlation techniques as applied to broad regional monitoring', Bulletin of the Seismological Society of America, vol. 104, no. 6, pp. $2768-2781$.

Spottiswoode, S \& Milev, A 1998, 'The use of waveform similarity to define planes of mining-induced seismic events', Tectonophysics, vol. 289, no. 1, pp. 51-60.

Spottiswoode, S, 2010, 'Mine seismicity: Prediction or forecasting?', Journal of the Southern African Institute of Mining and Metallurgy, vol. 110, no. 1, pp. 11-20.

Vaezi, Y \& Van der Baan, M 2015, 'Comparison of the STA/LTA and power spectral density methods for microseismic event detection', Geophysical Journal International, vol. 203, no. 3, pp. 1896-1908.

Vallejos, J \& McKinnon, S 2011, 'Correlations between mining and seismicity for re-entry protocol development', International Journal of Rock Mechanics and Mining Sciences, vol. 48, no. 4, pp. 616-625.

Waldhauser, F \& Ellsworth, W 2000, 'A double-difference earthquake location algorithm: Method and application to the northern Hayward fault, California', Bulletin of the Seismological Society of America, vol. 90, no. 6, pp. 1353-1368.

Wang, J \& Teng, T 1995, 'Artificial neural network-based seismic detector', Bulletin of the Seismological Society of America, vol. 85, no. 1, pp. 308-319.

White, B \& Whyatt, J 1999, 'Role of fault slip on mechanisms of rock burst damage, Lucky Friday Mine, Idaho, USA', SARES, vol. 99 , pp. 2.

Zhao, Y \& Jiang, Y 2010, 'Acoustic emission and thermal infrared precursors associated with bump-prone coal failure', International Journal of Coal Geology, vol. 83, no. 1, pp. 11-20. 